\title{
Case Report: Supra-Annular Mitral Valve Replacement in Infants with Congenital Mitral Valve Disease
}

\author{
Haoyong Yuan, MD, Zhongshi Wu, MD, Yifeng Yang, MD, Can Huang, PhD \\ Department of Cardiovascular Surgery, Second Xiangya Hospital, Central South University, Changsha, Hunan, China
}

\section{ABSTRACT}

Mitral valve replacement in infants is rare and causes a relatively high mortality, especially for patients under the age of 1. Supra-annular valve replacement is a viable technique for infants with a small valve annulus. Here, we report two infants who underwent mitral valve replacement via the supra-annular technique. The age and body weight of these babies were 2 months and 3 months and $4.1 \mathrm{~kg}$ and $4.7 \mathrm{~kg}$, respectively. Aortic mechanical valves were reversely implanted with a short segment of PTFE graft. The purpose of this strategy was to insert a larger mechanical valve and delay resternotomy. A two-year follow-up exam showed normal ventricular function without mechanical valve-related complications. This method is useful in treating neonates and infants.

Although the technique of mitral valve repair has improved over several decades, mitral valve replacement still is necessary at times. In neonates and infants with a small annulus, implantation of commercially available prosthetic valves in the annular position can be a challenge, and an age less than 1 year is a risk factor for early death [Selamet 2008]. Supra-annular mitral valve replacement (SMVR) is an alternative when a traditional annular implantation is not feasible [Sung 2008]. Herein, we report the cases of two patients, who underwent SMVR with a follow-up after two years.

\section{CASE REPORT}

Patient 1: A 2-month-old female infant with severe mitral regurgitation was diagnosed with short anterior and posterior mitral leaflets and thickened leaflet edges using transthoracic echocardiography (TTE). Noninvasive ventilation was administered for one month. Surgical treatment was performed with cardiopulmonary bypass $(\mathrm{CPB})$ at moderate hypothermia, and the mitral valve was exposed using incisions through the right atrium and atrial septa. The mitral valve repair initially was attempted, but it was unsuccessful. Thus, we were forced to replace the valve at a body weight of $4.1 \mathrm{~kg}$.

Received fune 7, 2019; accepted fune 24, 2019.

Correspondence: Can Huang, Department of Cardiovascular Surgery, Second Xiangya Hospital, Central South University, 139 Renmin Road, Changsha, Hunan, 410011, P.R. Cbina; +86-73185292133, fax +86-73185292133 (e-mail: buangcan413@csu.edu.cn).
The entire mitral valve was cut off, and the measured diameter of the mitral annulus was $16 \mathrm{~mm}$. A $20 \times 8 \mathrm{~mm}$ polytetrafluoroethylene (PTFE) vascular graft (W. L. Gore \& Associates, Inc) was sutured to the native valve annulus using interrupted, pledgeted, everting sutures. Thereafter, we inserted a reversed aortic mechanical valve (16-mm Medtronic, Inc) into the short segment PTFE tube graft and sutured the inflow margin of the graft onto the mechanical valve with a 4-0 prolene suture. The prosthetic leaflets were fixed on the graft, and this worked well. The patient was weaned from CPB without any issues five days after the operation. TTE showed good function of the mechanical valve without left ventricular outflow obstruction (MV PG $20 \mathrm{mmHg}$ versus $11 \mathrm{mmHg}$, before and after the operation, respectively). Heparin injection was administered every six hours at a dosage of $0.5 \mathrm{mg} / \mathrm{kg}$. Anticoagulation with warfarin was used to maintain the international normalized ratio between 2 and 2.5. After a follow-up period of 2 years, the patient was in good condition, and the TTE showed normal ventricular function without mechanical valve stenosis (MV PG $12 \mathrm{mmHg}$ ), regurgitation, or paravalvular leak (Table and Figure).

Patient 2: A 3-month-old, $4.7 \mathrm{~kg}$ female infant presented at age 2 months with dyspnea; she was under respirator-assisted ventilation for 17 days. TTE showed moderate mitral stenosis and severe mitral regurgitation with MV PG $24 \mathrm{mmHg}$. The mitral annulus was $19 \mathrm{~mm}$ in diameter. Mitral valve repair was attempted, but was not successful. A 19-mm aortic mechanical valve (Medtronic, Inc) was reverse implanted with a PTFE segment (24 $\mathrm{mm}$ in diameter, $1.5 \mathrm{~cm}$ in length) using the method previously described. After operation, the mechanical valve worked well, and the MV PG decreased to $12 \mathrm{mmHg}$. Anticoagulation treatment was administered as previously described. After a follow-up period of two years, the patient was in good condition, and the TTE showed normal ventricular function without mechanical valve stenosis (MV PG $12 \mathrm{mmHg}$ ), regurgitation, or paravalvular leak (Table).
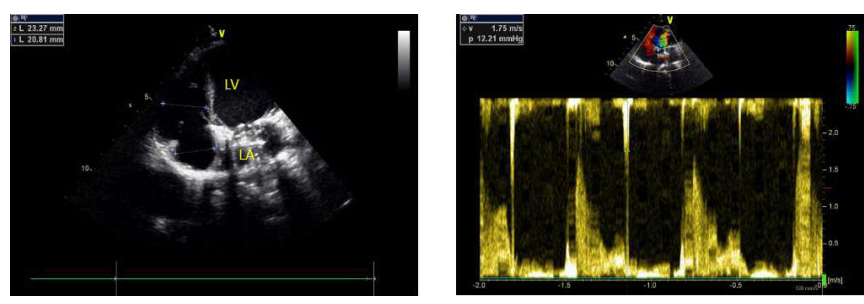

Figure. TTE images of two years follow-up 
Table. Patient Characteristics and TTE Findings Before/After SMVR

\begin{tabular}{lccc}
\hline & & Patient 1 & Patient 2 \\
\hline Age & & 2 months & 3 months \\
Weight & $4.1 \mathrm{~kg}$ & $4.7 \mathrm{~kg}$ \\
MV annulus size $(\mathrm{mm})$ & & 16 & 19 \\
Diagnosis & & MS & MR and MS \\
& Before & 35 & 40 \\
LA (mm) & 2 years later & 28 & 24 \\
& & & \\
MVPG (mmHg) & Before & 20 & 22 \\
& 7 days later & 11 & 12 \\
& 2 years later & 12 & 12 \\
\hline
\end{tabular}

$M V=$ mitral valve; $L A=$ left atrial; $M S=$ mitral stenosis; $M R=$ mitral regurgitation

\section{DISCUSSION}

Mitral valve replacement in infants is rare and causes a relatively high mortality, especially for patients under the age of 1 [Selamet 2008; Erez 2003]. Because of the small size of the annular mitral valve and unavailability of prosthetic heart valves, supra-annular mitral valve replacement was used to treat mitral valve diseases [Sung 2008]. Previously, the prosthetic heart valves were placed in a supra-annular position. Mattress sutures were placed onto the anterior annulus itself, followed by placement of sutures onto the anterior annulus, toward the posteriorly translocated supraannular MVR. Supra-annular valve replacement was associated with improved short-term survival and freedom from complete AV block [Selamet 2008]. However, supra-annular valve replacement might induce pulmonary hypertension caused by increased left atrial pressure, and a rigid mechanical valve must be sutured onto the friable annulus [Barker 2005]. Paravalvular leakage or other complications, such as late atrioventricular block may be seen [Giordano 2015]. Jong Soo Woo successfully implanted a 16-mm mechanical valve in the supra-annular position by suturing it into a short PTFE graft that was then sutured to the native annular in 2008 [Sung 2008]. Hiroaki Kawata used this method to insert a 16-mm mechanical valve with short-segment PTFE graft in a female neonate at 40 weeks [Yamauchi 2018]. We sutured 16-mm and 19-mm mechanical valves with PTFE grafts and implanted them into the two patients.

Reduced left atrial compliance caused by supra-annular MVR resulted in early pulmonary hypertension, and the pulmonary venous orifice is obstructed at times by the implanted prosthetic valve when this procedure is performed in small children [Giordano 2015]. Our patients were in good condition, and the TTE showed normal ventricular function without mechanical valve stenosis, regurgitation, or paravalvular leak on a 2-year followup. Jong Soo Woo considered that this technique could create a stagnant flow in the recess space around the valve in the left atrium and might enhance the probability of left atrial thrombus formation [Sung 2008]. In our patients, warfarin was used to maintain the international normalized ratio between 2 and 2.5; no thrombotic complications have been noted so far.

The frequency of MVR in infants and small children has decreased in the recent years. Nonetheless, some children in whom satisfactory repair cannot be accomplished will need MVR. Supra-annular valve replacement is useful in patients with smaller annulus where commercially available prosthetic heart valves cannot be implanted. In our recent experience, mechanical valve replacement with PTFE graft could avoid the obstruction of the pulmonary vein and may help avoid redoing an earlier MVR. This was useful in treating neonates and infants.

\section{REFERENCES}

Barker CL, Daubeney PE, Shinebourne EA. 2005. Complications of supra-annular mitral valve placement in infants. HEART. 91: e48.

Erez E, Kanter KR, Isom E, Williams WH, Tam VK. 2003. Mitral valve replacement in children. J Heart Valve Dis. 12: 25-29, 30.

Giordano R, Cantinotti M, Pak V, Arcieri L, Poli V, Assanta N, Moschetti R, Murzi B. 2015. Supra-annular mitral valve implantation in very small children. J Card Surg. 30: 185-189.

Selamet TE, Pigula FA, Berul CI, Lock JE, Del NP, McElhinney DB. 2008. Mitral valve replacement in infants and children 5 years of age or younger: evolution in practice and outcome over three decades with a focus on supra-annular prosthesis implantation. J Thorac Cardiovasc Surg. 136: 954-961, 961.

Sung SC, Chang YH, Lee HD, Woo JS. 2008. A novel technique of supra-annular mitral valve replacement. ANN THORAC SURG. 86: 1033-1035.

Yamauchi S, Iwai S, Tominaga Y, Kugo Y, Hasegawa M, Kayatani F, Takahashi K, Aoki H, Kawata H. 2018. Supra-Annular Mitral Valve Replacement in an Infant With Infective Endocarditis. ANN THORAC SURG. 105: e27-e29. 